\title{
Using machine learning to perform early diagnosis of autism spectrum disorder based on simple upper limb movements
}

\author{
Mohammad Wedyan ${ }^{\mathrm{a}, *}$, Adel Al-Jumaily ${ }^{\mathrm{a}}$ and Alessandro Crippa ${ }^{\mathrm{b}}$ \\ ${ }^{a}$ Faculty of Engineering and Information Technology, University of Technology Sydney, Sydney, NSW, Australia \\ ${ }^{\mathrm{b}}$ IRCCS Eugenio Medea, Scientific Institute, Bosisio Parini, Lecco, Italy
}

\begin{abstract}
Autism like other diseases requires early cure in order to magnify the remedy's results. The impact of Autism Spectrum Disorder (ASD) is embodied in the following things: the inability for kids to interact with other people, the difficulty in socialize with others, speaking after a long time comparing with other kids, lack of eye contact with other. Such activities are utilized for the resolution regarding diagnosing ASD. For instance, kids shift their upper limb before other activities and such moving considers as an indicator to decide whether such children suffer from autism. The current paper checks diagnosing autism that simply depends on altering upper-limb for kids between two to four years old that depends on carrying out certain mechanisms and machine learning. Such study utilized a Linear Discriminant Analysis (LDA) method to elicit features and, the Support Vector Machines (SVM) in order to categorize thirty kids i.e. categorizing around fifteen autistic kids out of fifteen normal children by analyzing kinematic information that is collected from implementing simple task. However, such study achieved an optimal precision categorization of $100 \%$ as well as $93 \%$ of intermediate precision. Such findings provide more clues for simple upper-limb movement that can be utilized in order to precisely categorize the kids who suffer from low-functioning autism.
\end{abstract}

Keywords: Autism, Kinematics, Linear Discriminant Analysis, Upper Limb, Early Autism Diagnosing, Machine Learning

\section{Introduction}

Autism defines as complex neurological disturbance. It considered as a portion of a group of disturbances that is known as autism spectrum disorders, and it manifests in the life of human $[1,2]$; such thing means that symptoms of autism disease differ from person to another according to the case of autistic child. In general, it ranges from mild to severe abnormal attitudes.

ASD is considered as one of the highest increasing developmental disorder in kids [3]. The statistics [4, 5] illustrate that autism ratio is increasing for clearing that the autism ratio in the 1970s was 1/10000 and in 2012 the percentage increased to $1 / 68$. Figure 1 indicates

\footnotetext{
*Corresponding author. E-mail:

MohammadOmar.M.Wedyan@student.uts.edu.au.
}

how autism diagnosis increased significantly from the 1970 s to 2012. Moreover, the percentage of autistic boys surpasses girls' percentage.

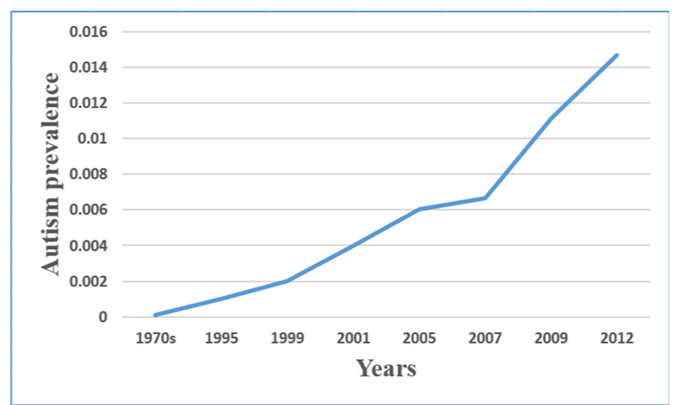

Fig. 1. Autism rate from the 1970 s until 2012 [1]

Autism is difficult to diagnose by medical doctors with support from physical, occupational and speech therapists since there is no medical test, doctors look 
at the child's behavior and development to make a diagnosis [6-8]. The diagnosis of autism is based on the child's failure to develop good social interaction, impairments in communication, poor eye contact, delayed speech and restricted repetitive and stereotyped pattern [6, 9-13]. Currently, the highest quality level of standard for the diagnosis of autism [14]:

- Clinical judgment of signs and with semistructured play-based behavioural observations.

- Standardized interviews or questionnaires for autism child family.

Begun to search the predictive value of neurobiological as well as behavioural measures in autism spectrum disorder in order to identify a well-defined phenotype of individuals and possibly to enable a computer-aided diagnosis perspective.

Diagnosing autism in early childhood enhances the opportunities for obtaining the best curing remedies. Actually the key factors that help the experts in revealing autism based primarily on noticing and are manifested in speaking after a long time, inability to contact with other, inability to develop normal social relationships [1]. The trials to detect the early signals neither stop nor try to attain early autism detection. The indicators that manifest whether the child has autism or not are embodied in the child's movement i.e. he/her ability either to walk or to move his/her upper limb [15]. Indeed, such movement indicators are extremely important since it aids in determining the early autism indicators in order to prevent any problem exacerbation in the future. Furthermore, such indicators might be measured $[14,15]$.

The effect of the fast development of technology especially in robotics and interactive software in the last few years is offering earlier and more accurate diagnoses of the Autism. In addition, it is giving costconscious diagnoses and treatment. Besides the reduced cost, the main advantage of utilizing these technologies can focus on one behavior which is hard even for a specialist and trained professional to perform that [16]. Also, technologies can help them to increase the in physical activity for children with autism because often do not engage in healthy levels of physical activity [17].

\section{Autism and kinematic data}

The relationship between the autism and their movement's measurements has started considerably in the last decade ago [18] in many studies. These studies showed that an autistic child ability is different from a typical child [1, 14, 15, 19-21] these differences were huge in quantity and manner [18]. Furthermore, these differences are increasing as age developed [18]. In Addition, Many autistic children also have atypical movement patterns, such as asymmetric gait or toe walking [22]. It is widely known to specialists that there is a flaw of overall movements function in autistic children [23-27] but have not investigated or studied, and there are few empirical papers, with the exclusion of repetition habits, is not among its diagnostic criteria [25-27].

Motion analysis is based on kinematic gait data [22, $25,28]$ and upper limb data $[1,14,15,19,20]$, autismspecific movement signs which, being obtained before the development of language, and being assessed quantitatively [14, 15]. In [29] the participants are adults, the participant's autistic arm movements differed from typical participants on measurements of the jerk, velocity, and acceleration Also, in [30] they concluded that kids with autism movements are differentiated in the endpoints they referred to the reason was a cerebellar disturbance. These differences in an upper limb movement's disorder in autism and typical children can help specialists as a future clinical measure. In [31], the study finds functional organization dissimilarities in the motor cortex in autistic kids compared with typical kids. These variations in the segregation and size of primary motor cortex parcels, therefore, the functional sub networks in the motor control system may be changed in an autistic child.

Finally, the aim of this paper is to do more investigation of the relationship between autism and kinematic movement and especially upper limb movements and try to reach high classification precision via using accurately recorded tracking data and different extracting and classification techniques.

\section{Related work}

There are a little research that investigated the relationship between the upper limb movements and for children with autism. But they reached an important conclusion, which is there a little upper limb motioning might be utilized to precisely categorize autistic children who suffer from low-functioning.

In [14] Fisher discriminant ratio was used to select the features from data then SVM was also used to classify preschool-elderly participants with autism have been 
in comparison with 15 typically developing participants who had been matched by using mental age the result show classification accuracy of $84.9 \%$.

In [32] paper evaluates diagnosing autism that builds on moving the children's arms aged between 24 and 48 months by doing some safe tasks and machine learning to classify them. The method that such study used is both linear discriminant analysis in order to select the features and support vector machines for classifying thirty children such study selected 15 autistic kids out of 15 non-autistic kids by testing the gathered data that are gathered after doing a simple, safe, and exciting task. Finally, the outcomes of such search have accomplished an optimal classification accuracy of $100 \%$ and the average classification accuracy of $93.8 \%$. Such results provide more evidence that simple brachium motioning that might be used in diagnosing of autistic children precisely.

Wedyan et al. reported their studies in $[1,19,20]$ upper limb is valuable in enhancing the medical practice of ASD diagnosing. Consequently, such a thing might enhance the diagnosis with computer assistance. They used LDA as eliciting features, and SVM as a classification method. Furthermore, such outcomes provide a view of practicable motor autism marks.

In this study [15] classifying approach SVM to upper limb movements was used. To recognize special features in "reach-and-throw" motion. Ten autistic kids with preschool age kids and ten non-autistic kids doing the same tasks were examined. The SVM approach shows that be able to differentiate the two collections: the result was achieved $100 \%$ and $92.5 \%$ with a soft margin algorithm and with an additional conservative one, respectively. These outcomes have acquired with a "radial basis function kernel," and they suggested that a "non-linear analysis" is perhaps wanted.

\section{Method}

This section provides a description of children, mechanisms concerning information gaining, little training, properties deriving by utilizing LDA, categorization by the assistance of SVM, and assessment depends on performance. In fact, the performances in such paper regarding the classification for ASD children are contrary to typically growing children (TG) i.e. normal children. Such categorization was conducted by utilizing SVM. The overall amount of kinematic information was (17 criteria) LDA was executed in order to derive properties from raw kinematic in- formation. After that, the extracted features $(\mathrm{EF})$ were divided into two groups; the first group was an experimenting group that contains a signal subcategory of the original EF, and the training group that contains the rest of groups of the EF executed by utilizing machine educating methods, SVM. The schema that explains the full method is manifested in Figure 2.

\subsection{Participants}

The comparison was made between fifteen of preschool-aged autistic children with the same amount of normal children who have the same rational age. The ages of kids are ranged between 2-4 years. The participant's data is manifested in Table 1. IRCCS Eugenio Medea - Scientific Institute for Research evaluated IQ and mental age by adopting "Griffiths Mental Development Scales" [33]. Indeed, the little finding that emerges in Griffiths measurement clarifies that entrants, with low-functioning as a standard clinical mechanism, aged between one to two years old has been demonstrated to be a precise indicator of impairment at school age [34]. However, the whole kids had natural or corrected-to-natural sight.

The kids in the ASD room were enlisted at IRCCS Eugenio MedeaScientific Institute for research for a period exceeds one and a half year. Early diagnosis was conducted for each kid in the medical center as the mentioned standard notified in "Diagnostic and Statistical Manual of Mental Disorders-IV-TR" [35] by a practitioner specialized in children neuropsychiatry.

The diagnosis regarding the child's psychological status is assured by direct observation and speech with each child's family. TG class compromised domestic kids and from nursey to be reasonably the same age to the clinical children from the normally developed people. After that, it was inserted as a contrastive class. In any case, TG entrant conform to mental age, in addition to the assumption that mental age normally portends the capacity to perceive task instructions, utilizing appropriate techniques, and prohibiting unsuitable responses [36]. TG sharers do not have any prior history concerning social interaction dilemma, development disorder, or health troubles concerning central nervous system complications. In order to obtain both more information and ethics agreement return to resources [14].

\subsection{Data Acquisition}

The main usage for optoelectronic system is embodied in registering the motion information. 3D motion 


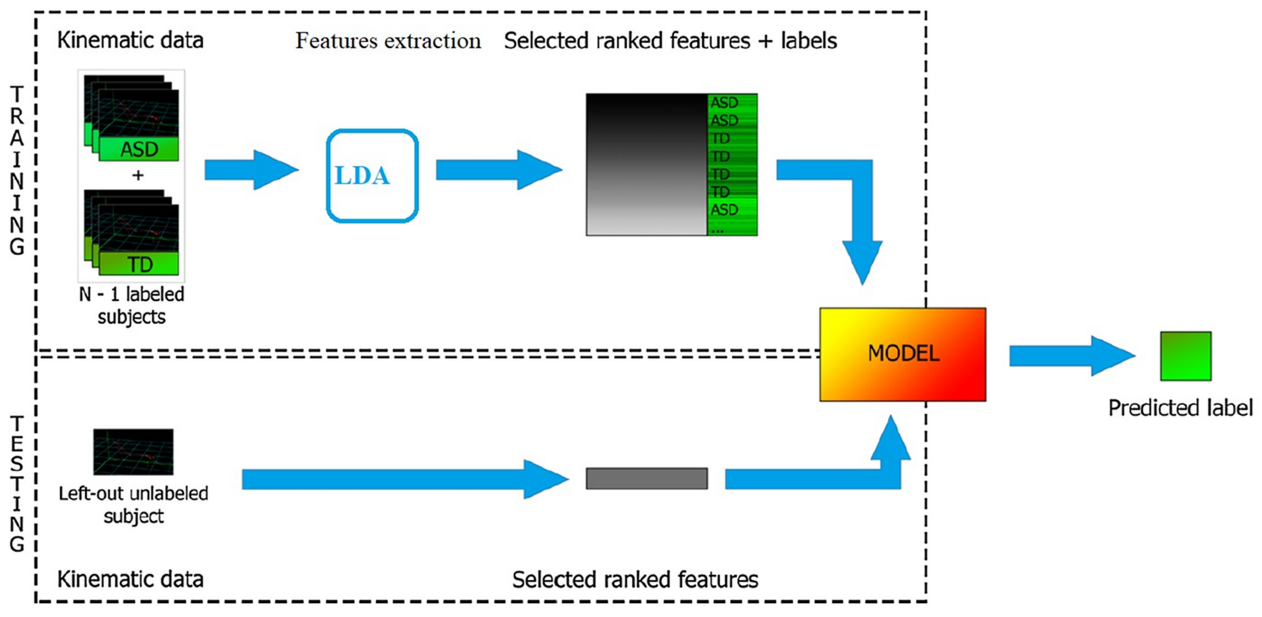

Fig. 2. The diagram that illustrated all steps in this paper.

Table 1

Participant Characteristics

\begin{tabular}{lll}
\hline & Autism Spectrum Disorder (ASD) & Typically Growing (TG) \\
\hline Number & 15 & 15 \\
male:female & $12: 3$ & $13: 2$ \\
age (years) & between 2 and 4 years & between 2 and 4 years \\
\hline
\end{tabular}

information is listed through 8 infrared motion cameras that are inserted at four each part from the children. Moreover, little passive markers were located on both ulnar and radial parts of the children's wrists, the back of their hand, and on the fourth and fifth hand's bones. Also, four markers were inserted on the box's limits beneath the purposed area while two of them inserted on the ball. Firstly, the whole amount of motion data is subjected to prior processing by Matlab while motion distribution and variables approximations were gathered by the software [14]. The motions (manifested in Figure 3 and mentioned in Table 2) were divided into two groups, sub-motion one that was illustrated as adopting the required movement to access to the ball and insert it for supporting it; sub-motion two is illustrated as the ability to move the ball from its original place until reaching to the box opening place where the ball was to be dropped.

The statistics regarding a category of dependent amounts were collected. Such sub-motions are submotion 1, which contain; motion units, the whole motion period, peak speed, the date of peak speed, peak rapidity, date of peak increasing, peak decreasing, and the date of peak slowing. In addition, sub-movement 2 that contains, motion units, the whole amount of motion period, peak speed, time of peak speed, peak ra- pidity, date of peak rapidity, peak speed, date of peak speed, date of peak rapidity, peak slowing, date of peak slowing, and carpal angle, such 17 motion information are used as input properties for categorization system. Table 2 indicates the analysis of kinematic information whether (mean and standard deviation) values for the children's group. The definition of motion unit is the transferring from rapidity phase until slowing phase for an amount that surpasses 10 millimeters for each second. It happens when an accumulated speed exceeds twenty millimeters for each second, rising or falling $[14,37,38]$.

\subsection{Task}

The mission is embodied in setting the children on modifiable height chair that was adjusted according to the sharers body before desk while the supervisor sat on the fronting side of the desk. Moreover, both parents were existed. The assessment initiates when the sharers' hands, placing at a determined site, stay away from the ball around twenty centimeters. The study mission is consisted of picking the tiny rubber ball that was placed for helping them (see Figure 3, a); the children noticed movement before children fallen it in an open box. The open box (see Figure 3, b) was inserted in a 
clear square box (see Figure 3, c) and was sufficiently huge without the need of soft movements. Each child has executed ten trials that were distributed as follows five consecutive trials on another part. The order of experiment's groups was balanced among children. The mission is initially carried out by the examiner in order to clarify the mission request like holding the ball and tossing it inside the box). In case the lack of any actual mark. Practice trials, the amount that is personally diverse. After that, it was handed to the children prior registration to emphasize the children's recognition to the trial. The children were permitted to take a rest during the trial upon their request. The experiment's task was enough simple and interesting $\mathrm{n}$ order to guarantee obtaining the sufficient motivation and adherence from the children's part across groups.

\subsection{Feature Extraction}

In fact, such paper utilized Linear Discriminant Analysis (LDA) that defines as a properties' derivation technique that is mostly utilized to raw information in order to derive features. The technique's significance lies in getting rid of abundant and unrelated properties. Consequently, classification of new examples will be more precise [39]. The more the amount of features enhances, the more the computational costs enhances in order to resolve such matter it is vital to search for a method to decrease the dimensionality of the given raw information or properties (features). The benefits of feature derivation are higher preferential force and domination that are very applicable when they are done without surveillance, but from the other side information loss explainability and changing might be costly [40].

LDA defines as a well-known information mining algorithm that can be utilized for monitored and unmonitored educating [41]. The benefits of LDA lies in the resolution that can be obtained through resolving popularized intrinsic technique. Thus, LDA permits a rapid and huge processing of information samples [42]. Their methods are utilized for classification and decreasing information dimensionality [43]. Such study utilized LDA for generating properties through decreasing information dimensionality.

The LDA mechanism depends on revealing the "projection hyper-plane" that decreases the intergroup variation and increases the gap among the dual categories [44] as manifested in Figure 4. Such hyperplane can be utilized for various goals like categorization, magnifying dimensionality, and most impor- tantly for the revelation of the given properties importance [41].

Dual categorization information utilizes LDA while multi-group employs Multiple Discriminant Analysis (MDA)

The equation of LDA is [45]

$\mathrm{DS}=\mathrm{W}_{1} \mathrm{C}_{1}+\mathrm{W}_{2} \mathrm{C}_{2}+\ldots+\mathrm{W}_{n} \mathrm{C}_{n}$

While

$\mathrm{DS}=$ discriminant score, $\mathrm{W}=$ discriminant weights, and $\mathrm{C}=$ independent variables

The above mentioned study [14] utilized Fisher's discriminant ratio FDR that considers as an effective property chosen method [46] that chose seven properties out of seventeen properties, the chosen properties were, overall period, ; delta wrist angle; amount of motion units, the date of peak slowing, peak increasing, date of peak speed, and peak speed.

LDA might be achieved through magnifying FDR ratio that is between group distinctions in general and group distinction for the training group in particular. Employing LDA for both groups, the information will be converted to one-dimensional subspace, and for multi-group (C). After that, the decrease dimensionality is (C-1) [47]. Lastly, the division of ASD and TG after implementing LDA is manifested in Figure 5.

\subsection{Support Vector Machine model (SVM)}

SVM is an educating approach that is utilized in order to determine labels to matters. Therefore, it is named as learning by practicing instances; it is monitored educating algorithm that used for retraction and categorization problem [48-50]. The standard of SVM work based on analyzing a statistically practicing group and looking for appropriate role through a set of roles. The optimal role lies in decreasing a defined risk (empirical risk). The risk is conditioned on both the selected set of roles complexity and the practicing group [51]. It considers as a widespread categorization method that is employed in order to obtain the optimal educating precision. Consequently, such paper utilizes SVM as the categorizer (classifier) method. However, the details concerning SVM method are completely mentioned in [52]. Such work used both the SVM and a linear kernel (shown in Figure 6).

Training data form is:

$$
\left\{x_{i}, y_{i}\right\} \text { where } i=1 \ldots L ; y_{i} \in\{-1,1\} x \in R(1)
$$

Given a training set of instance-label pairs $\left(x_{i}, y_{i}\right)$

$\mathrm{L}$ means training point

D means dimensional 

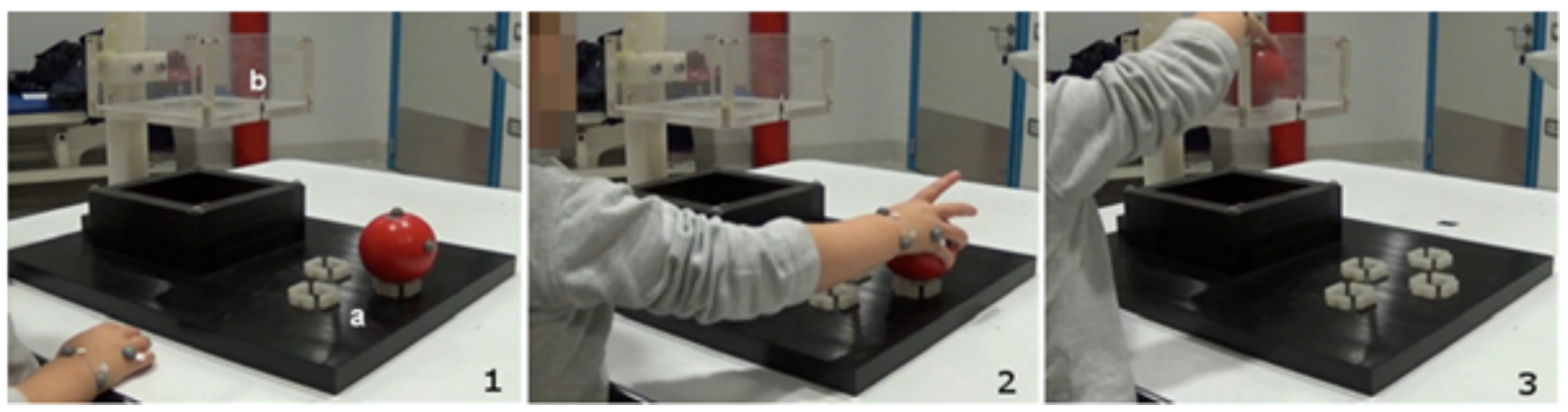

Fig. 3. Illustrates the trial task steps: 1$)$ rubber ball located above support (see $1, a)$, the box $(1, \mathrm{~b})$ was placed inside a transparency box and was big enough to require smooth motion. 2) The child catches a small rubber ball; before he is fallen it in a box (3). The goal zone is see-through plastic to allow seeing through it. Four markers are located on the basket, on the ball, and on each wrist [14].

Table 2

Shows kinematic data analysis (Mean and Standard Deviation) values of the Two collections of Kids appeared in the paper (ASD Versus TG).

\begin{tabular}{|c|c|c|c|c|}
\hline & $\begin{array}{l}\text { ASD } \\
\text { (Mean) }\end{array}$ & $\begin{array}{l}\text { ASD } \\
\text { (Standard Deviation) }\end{array}$ & $\begin{array}{l}\text { TG } \\
\text { (Mean) }\end{array}$ & $\begin{array}{l}\text { TG } \\
\text { (Standard Deviation) }\end{array}$ \\
\hline \multicolumn{5}{|l|}{ Submovement 1} \\
\hline Movement units & 1.91 & 0.62 & 1.70 & 0.37 \\
\hline Total movement duration & 0.69 & 0.14 & 0.66 & 0.12 \\
\hline peak velocity & 0.46 & 0.12 & 0.59 & 0.17 \\
\hline Time of peak velocity & 0.34 & 0.07 & 0.31 & 0.04 \\
\hline Peak acceleration & 3.18 & 0.93 & 4.26 & 1.52 \\
\hline $\begin{array}{l}\text { Time of peak accelera- } \\
\text { tion }\end{array}$ & 0.21 & 0.07 & 0.16 & 0.05 \\
\hline Peak deceleration & -3.59 & 1.28 & -3.93 & 1.44 \\
\hline $\begin{array}{l}\text { Time of peak decelera- } \\
\text { tion }\end{array}$ & 0.47 & 0.08 & 0.44 & 0.06 \\
\hline \multicolumn{5}{|l|}{ Submovement 2} \\
\hline Movement units & 3.45 & 1.78 & 1.76 & 0.39 \\
\hline Total movement duration & 1.35 & 0.44 & 0.79 & 0.15 \\
\hline Peak velocity & 0.61 & 0.15 & 0.76 & 0.16 \\
\hline Time of peak velocity & 0.41 & 0.14 & 0.31 & 0.05 \\
\hline Peak acceleration & 3.85 & 1.13 & 5.85 & 1.94 \\
\hline $\begin{array}{l}\text { Time of peak accelera- } \\
\text { tion }\end{array}$ & 0.23 & 0.20 & 0.13 & 0.04 \\
\hline Pick deceleration & -3.29 & 1.15 & -4.27 & 1.88 \\
\hline $\begin{array}{l}\text { Time of peak decelera- } \\
\text { tion }\end{array}$ & 0.75 & 0.24 & 0.51 & 0.11 \\
\hline Wrist angle & -4.25 & 16.34 & -25.0 & 12.40 \\
\hline
\end{tabular}

The hyper plane is described via $w \cdot x+b=0$

w means normal to the hyper plane $\frac{b}{\|w\|}$ is the vertical length from the hyper plane to origin. Referring to the graph in Figure 6 The training data can be written as follows

$$
\begin{aligned}
& x_{i} \cdot w+b \geqslant+1 \text { for } y_{i}=+1 \\
& x_{i} \cdot w+b \leqslant-1 \text { for } y_{i}=-1
\end{aligned}
$$

$\mathrm{w}$ also $\mathrm{b}$ are learning data.

Then these two equations combine in (4):

$$
y_{i}\left(x_{i} \cdot w+b\right)-1 \geqslant 0 \forall_{i}
$$

The planes $\mathrm{H} 1$ and $\mathrm{H} 2$ can be written by:

$$
\begin{array}{ll}
x_{i} \cdot w+b=+1 & H_{1} \\
x_{i} \cdot w+b=-1 & H_{2}
\end{array}
$$

\subsection{Classification Algorithm Evaluation}

This study tackles binary categorization information that considers the most prevalent categorization mission and defines that as the matter is to be divided into merely one group of two non-interrelated groups [54]. Consequently, there are a lot of diverse categorization assessments that deem significant in gauging the ad- 


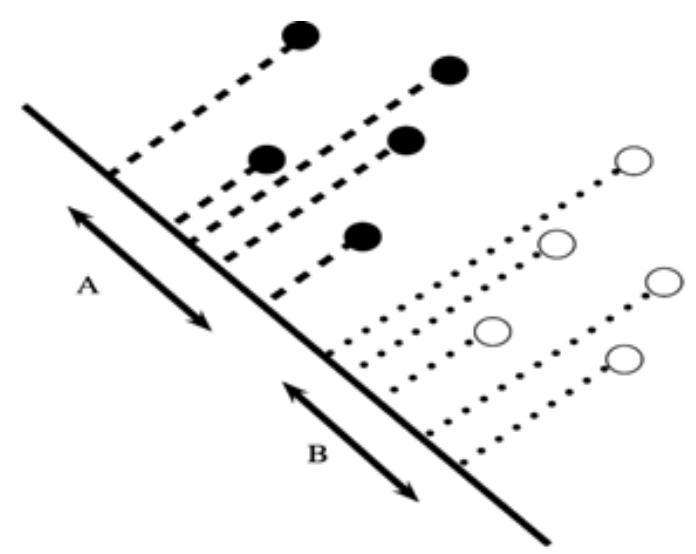

Fig. 4. The "projection hyper-plane" that lessen the inter-class difference and expand the space between the binary groups [41]

vantage of categorization and learning algorithm [55]. The aim of Cross Validation (CV) technique was conducted to evaluate the accomplishment of categorization algorithm. Generally, CV technique is building on dividing the whole database into two integral subgroups-namely training and experimenting groups. In general, the training group is major than the experimenting group. The definition of training group is a group of properties that re connected to a label, it is utilized in order to execute the training of the categorizer. The experimenting group defines as a group of properties that are not linked with a label and utilized in order to execute categorizer's efficiency through implementing various information's division. After that, several times of information can be executed.

An example of $\mathrm{CV}$ is manifested in Leave-one-out (LOO) cross-effectiveness, the primary utilize of it is to examine the research effectiveness since it considers as a neutral evaluation of the flaw likelihood. The technique of LOO depends on the standard of dividing the whole into $\mathrm{N}$ sub-groups, one of them is utilized for experimenting while the rest of the group $(\mathrm{N}-1)$ is utilized for categorizer training. Therefore, per each circle, N-1 sub-groups are utilized for training group while the rest of the item is utilized for experimenting, and so forth. Thus, it is preferable for the amount of periods to implement number $\mathrm{N}[14,56]$.

In dual categorization information, there are three most common utilized measurements, such as the Sensitivity (recovery), Specificity, and precision. The aim of utilizing such measurements lies in their importance to check the categorizer validity that depends on four counts. They are truly categorized and refer to the group called (correct positives), untruly categorized and refer to the group called (wrong negatives), untruly categorized and do not refer to the group (wrong negatives) [54]. Such measurements were counted to measure the categorizer achievement.

Sensitivity $=$ correct positives $/$ (correct positives + wrong negatives)

Specificity $=$ correct negatives/ (wrong positives + correct negatives)

Accuracy $=$ (correct positives+ correct negatives)/(correct positives + correct negatives + wrong positives + wrong negatives)

Sensitivity in such study defines as the average of a truly classified type in the ASD collection. Quality defines as the average of truly classified types in the (TG) class. Lastly, the precision of classification that defines as the average of truly classified types in both ASD and TG collections [57].

Sensitivity $=($ The count of ASD children that truly classified ASD children)/ (Count of all ASD children)

Specificity $=($ The count of TG children that truly categorized)/ (Count of all TG children)

Accuracy $=($ The count of children that truly categorized)/ (Count of all children)

Indeed, the overall machine learning stages whether (derivation or categorization) were utilized on the Matlab platform (version R2017a). Such paper particularly utilized properties of the toolbox of Matlab 2017 to achieve the classification algorithm.

\subsection{Evaluation}

The key factor for evaluating the performance of classification, as mentioned above, is precision. Such measurements for all circles were calculated by utilizing 5-fold CV. However, in every fold a single group is chosen as the testing group while the remaining groups were used as a training group. The intermediate measurement regarding the fifth divisions was utilized for each round. Furthermore, in order to clarify the empirical results statistical analyses were employed. As stated previously, the experiments were created on the gathered information.

\subsection{Results}

SVM code [58-60] was utilized in such paper that divides the group properties haphazardly into both groups, namely training and experimenting groups. SVM algorithm was occurred for five times $(\mathrm{N}=5)$, it has chosen distinct six children as experiment group while the remaining twenty-four children as a train- 


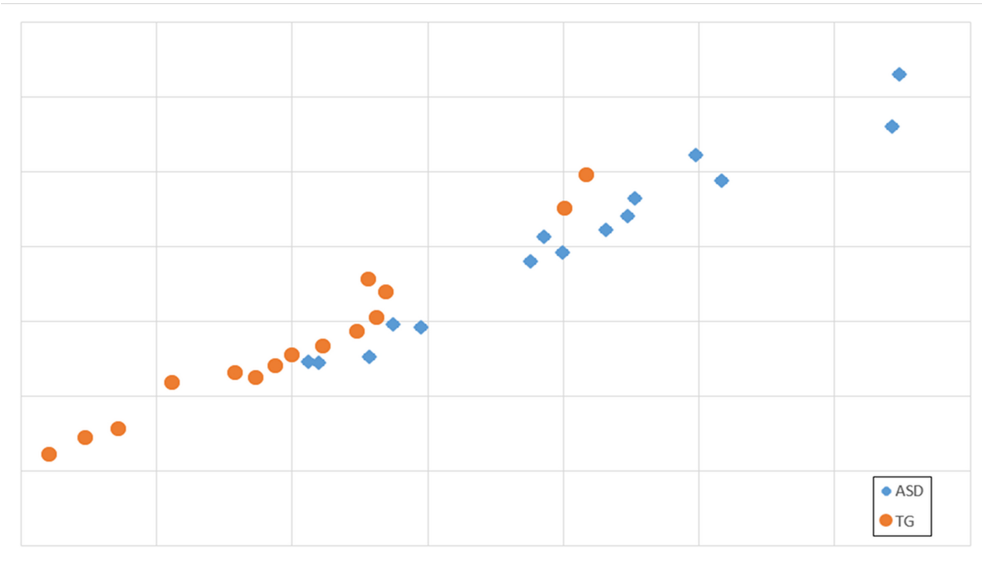

Fig. 5. shows the distribution of ASD and TG after performing LDA.

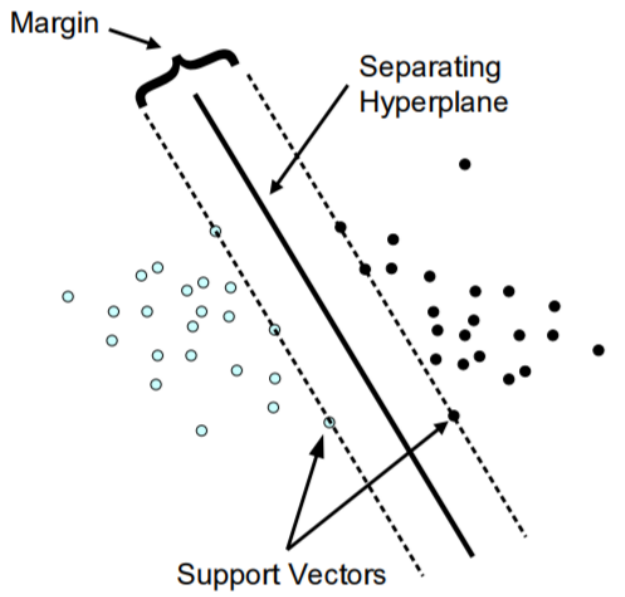

Fig. 6. Linear separable [53]

ing group. The SVM permitted to successfully classify children by counting the whole mean categorization, and the following conclusions emerged, intermediate sensibility is $91.0 \%$, while intermediate quality is $98.1 \%$ and intermediate precision is $93.8 \%$.

This finding generally consistent with various studies. For instance, Alessandro et al. in [14] which had tackled a proof-of-concept study in order to define whether a hand motion might be utilized to categorize precisely low-functioning autistic children, and they achieve $84.9 \%$ While Wedyan et al. in [1] their study concentrated on defining if a simple upper-limb motion might be utilized to categorize infants at high risk of autism as well as comparing them with infants at lowrisk of autism, and they achieved $93.8 \%$ precision. Table 3 and Figure 7, Figure 8, and Figure 9 indicate that the increasing that was accomplished after employing
LDA instead of Fisher discriminant ratio (FDR) utilized the same categorization tools precision, sensibility, and precision, successively.

The column charts in Figure 7, Figure 8, and Figure 9 manifest the increasing of precision, sensibility, and quality after using LDA rather than FDR. The utmost increasing was in the quality that achieved $9 \%$. After that, the precision that achieved $8.9 \%$. Lastly, the sensibility increasing that achieved $8.8 \%$ comparing with FDR algorithm. In general, it is evident that LDA algorithm has an obvious selection.

\section{Discussion and Future Work}

Discovering and diagnosing autism at early stages will help the early involvement that magnifies the remedy's results. ASD diagnosis resolution depends on kids' motion to their upper limbs before any other activities. The current study revealed the diagnosing of the ASD by depending on simple motioning to upperlimb for children whose ages ranged between two to four years by carrying out certain procedures and algorithms. LDA was utilized to derive properties from the gathered sub-motions that considered more distinctive for the ASD comparing with TG. Property generation was executed by employing LDA mechanisms for the purpose of searching for significant group variation that depends on the kinematic information. However, the reason behind using it refers to various goals like information classification and decreasing information dimensionality [32], such study adopted LDA for generating properties decreasing information dimensionality. 


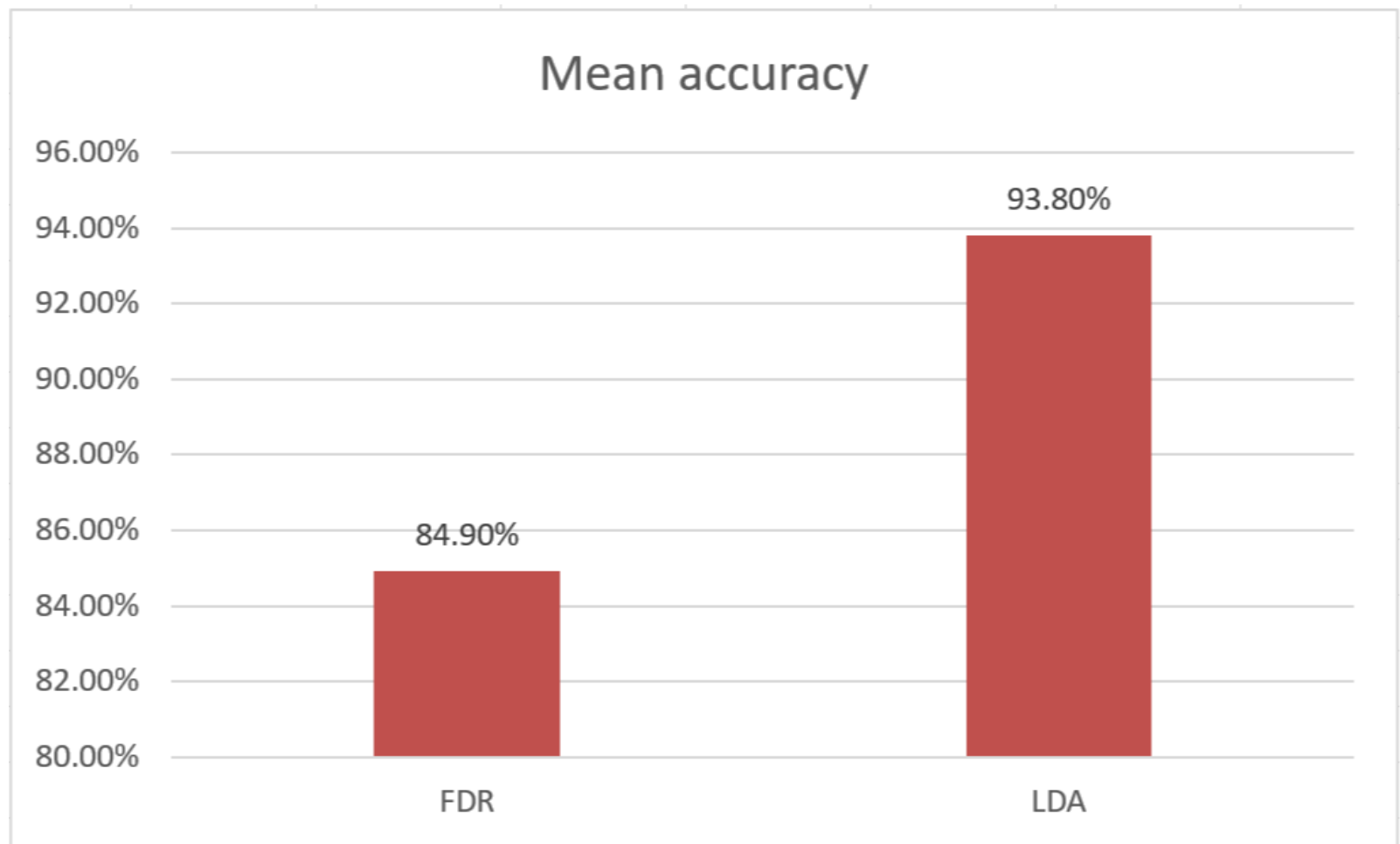

Fig. 7. Shows the mean accuracy enhancement after applied LDA comparing to FDR.

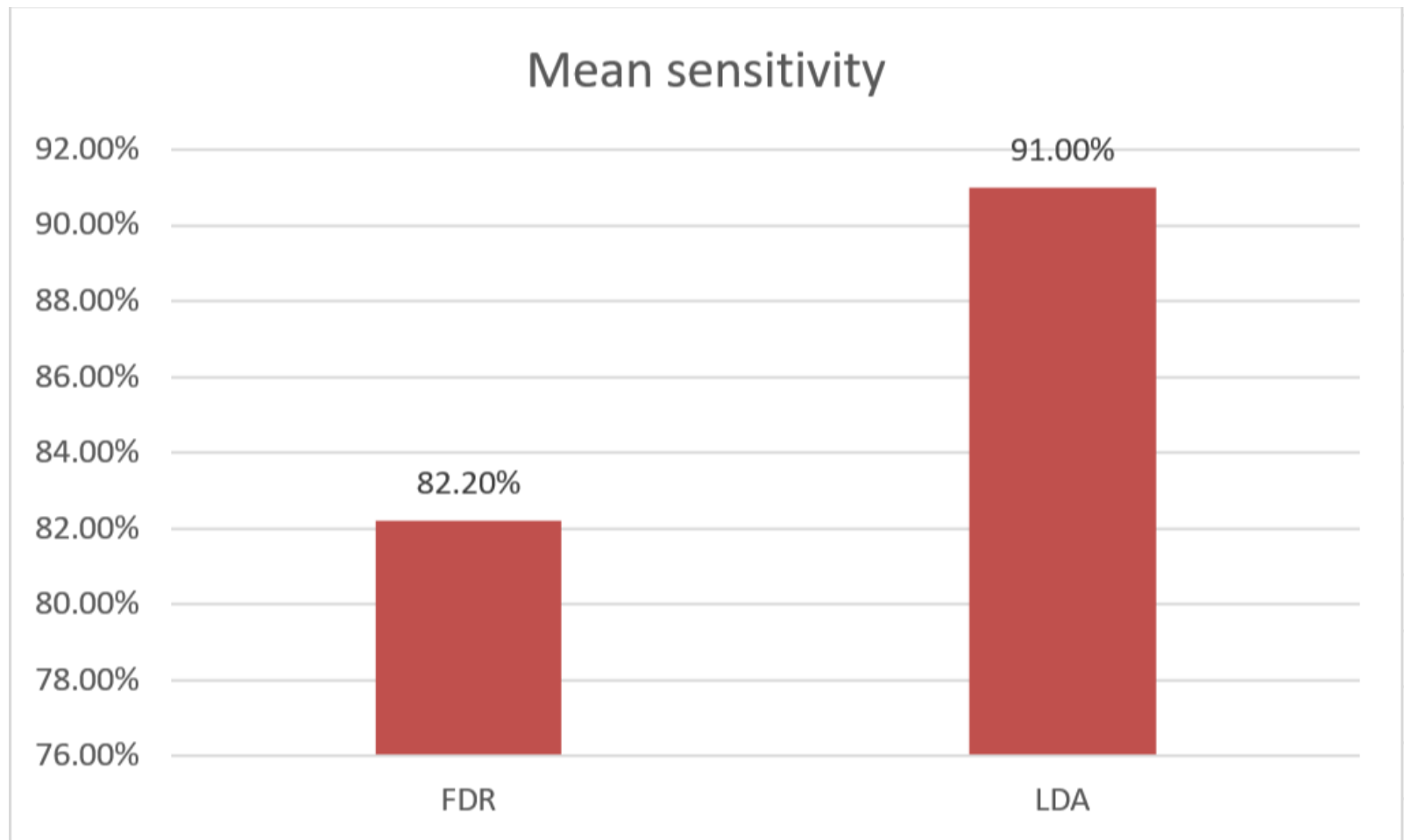

Fig. 8. Shows the mean sensitivity enhancement after applied LDA comparing to FDR. 
Wedyan, Al-Jumaily and Crippa / Using machine learning to perform early diagnosis of autism spectrum disorder based on simple upper limb movements

Table 3

Shows different studies and their results using upper limb data set.

\begin{tabular}{llllc}
\hline $\begin{array}{l}\text { Study } \\
\text { (IRCCS Eugenio Medea } \\
\text { dataset) }\end{array}$ & $\begin{array}{l}\text { The algorithm was ap- } \\
\text { plied to select or extract } \\
\text { features }\end{array}$ & Mean accuracy & Mean sensitivity & Mean Specificity \\
\hline$[14]$ & FDR & $84.9 \%$ & $82.2 \%$ & $89.1 \%$ \\
{$[1]$} & LDA & $78.5 \%$ & $66.7 \%$ & $72.5 \%$ \\
{$[19]$} & LDA & $75.0 \%$ & $76.47 \%$ & $73.33 \%$ \\
Proposed Work & LDA & $93.8 \%$ & $91.0 \%$ & $98.1 \%$ \\
\hline
\end{tabular}

\section{Mean Specificity}

\section{$100.00 \%$}

\section{$98.00 \%$}

$96.00 \%$

$94.00 \%$

$92.00 \%$

$90.00 \%$

$88.00 \%$

$86.00 \%$

$84.00 \%$

$89.10 \%$

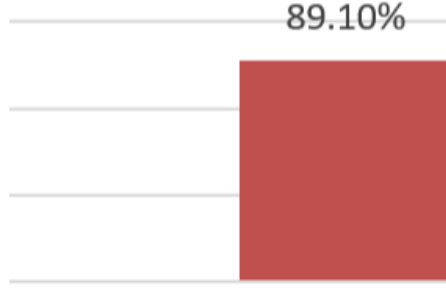

FDR

\section{$98.10 \%$}

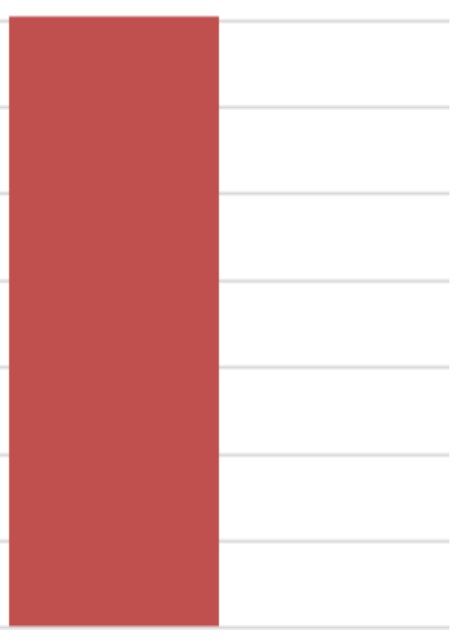

LDA

Fig. 9. Shows the mean Specificity enhancement after applied LDA comparing to FDR.

In such study, SVM was used as a categorization algorithm. SVM considers as a learning model that determines labels to matters. Therefore, it is named as learning by training instances. The SVM permitted classifying kids successfully by counting the overall mean categorization and achieving the following findings; average sensitivity reaches to $91.0 \%$ while average quality is $98.1 \%$ and intermediate precision is $93.8 \%$.

Such study illustrates that such findings provide more clues that a simple upper-limb motion could be utilized to precisely categorize low-functioning autistic children. Furthermore, such kinematic analyzing of simple upper-limb motion can dependably define early (3 years), low-functioning autistic children. The considerable predictive amount of LDA deriving process might sound important for enhancing the clinical training for diagnosing ASD. Therefore, enhancing the view of computer-aided diagnosis. In addition, the findings provide view of practical motor autism signals that might be useful for defining children subcategories clearly. Thus, decreasing the lack of coherence within the huge behavioral phenotype.

The upcoming work shall require urging a very large sample to reveal bigger potentially concerning differences. For instance, the sex variations in each of the TG and ASD sharers to generate considerable variations. Furthermore, the dire need to examine the man- 
ner of participants' reactions towards remedies in case if we want to improve a better remedy for sharers with ASD.

\section{Acknowledgments}

The authors would like to acknowledge Scientific Institute IRCCS Eugenio Medea in Italy for permitting the researcher to reach such kinematics information group.

\section{References}

[1] M. Wedyan and A. Al-Jumaily, Upper limb motor coordination based early diagnosis in high risk subjects for Autism, in: 2016 IEEE Symposium Series on Computational Intelligence (SSCI), IEEE, 2016, pp. 1-8.

[2] E.M. Rojas, M.R. Ramirez, H.B.R. Moreno, M.S. Caro, A.A. Garza and J.S.M. Palencia, Autism Disorder Neurological Treatment Support Through the Use of Information Technology, in: International Conference on Innovation in Medicine and Healthcare, Springer, 2016, pp. 123-128.

[3] C. Kanimozhiselvi, M.D. Jayaprakash and M.K. Kalaivani, Grading Autism Children Using Machine Learning Techniques, International Journal of Applied Engineering Research 14(5) (2019), 1186-1188.

[4] R. Guillén, C. Jensen and S. Edelson, A machine learning approach for identifying subtypes of autism, in: Proceedings of the 1st ACM International Health Informatics Symposium, ACM, 2010, pp. 620-628.

[5] N.I. of Mental Health, 2016. http://www.nimh.nih.gov/health/ statistics/index.shtml.

[6] E.A. Alsaggaf and S.S. Baaisharah, Directions of Autism Diagnosis by Electroencephalogram Based brain Computer Interface: A Review, Life Science Journal 11(6) (2014), 298-304.

[7] M.D. King and P.S. Bearman, Socioeconomic status and the increased prevalence of autism in California, American sociological review 76(2) (2011), 320-346, in California.

[8] M. King and P. Bearman, Diagnostic change and the increased prevalence of autism, International journal of epidemiology 38(5) (2009), 1224-1234.

[9] P.I. Markowitz, Autism in a child with congenital cytomegalovirus infection, Journal of autism and developmental disorders 13(3) (1983), 249-253.

[10] R.C.M. Philip and University of Edinburgh, Emotion processing in autism spectrum disorder, 2009.

[11] L. Zwaigenbaum, S. Bryson, T. Rogers, W. Roberts, J. Brian and P. Szatmari, Behavioral manifestations of autism in the first year of life, International journal of developmental neuroscience 23(2-3) (2005), 143-152.

[12] G. Dawson, J. Osterling, A.N. Meltzoff and P. Kuhl, Case study of the development of an infant with autism from birth to two years of age, Journal of applied developmental psychology 21(3) (2000), 299-313.
[13] P.J. Krantz and L.E. McClannahan, Social interaction skills for children with autism: A script-fading procedure for beginning readers, Journal of applied behavior analysis 31(2) (1998), 191-202.

[14] A. Crippa, C. Salvatore, P. Perego, S. Forti, M. Nobile, M. Molteni and I. Castiglioni, Use of Machine Learning to Identify Children with Autism and Their Motor Abnormalities, Journal of autism and developmental disorders 45(7) (2015), 2146-2156.

[15] P. Perego, S. Forti, A. Crippa, A. Valli and G. Reni, Reach and throw movement analysis with support vector machines in early diagnosis of autism, in: 2009 Annual International Conference of the IEEE Engineering in Medicine and Biology Society, IEEE, 2009, pp. 2555-2558.

[16] X. Liu, Q. Wu, W. Zhao and X. Luo, Technology-facilitated diagnosis and treatment of individuals with autism spectrum disorder: an engineering perspective, Applied Sciences 7(10) (2017), 1051

[17] M.N. Savage, T. Taber-Doughty, M.T. Brodhead and E.C. Bouck, Increasing physical activity for adults with autism spectrum disorder: Comparing in-person and technology delivered praise, Research in developmental disabilities 73 (2018), $115-125$.

[18] K.L. West, Infant Motor Development in Autism Spectrum Disorder: A Synthesis and Meta-analysis, Child development (2018).

[19] M. Wedyan and A. Al-Jumaily, Early diagnosis autism based on upper limb motor coordination in high risk subjects for autism, in: 2016 IEEE International Symposium on Robotics and Intelligent Sensors (IRIS), IEEE, 2016, pp. 13-18.

[20] M. Wedyan and A. Al-Jumaily, An investigation of upper limb motor task based discriminate for high risk autism, in: 2017 12th International Conference on Intelligent Systems and Knowledge Engineering (ISKE), IEEE, 2017, pp. 1-6.

[21] S. Forti, A. Valli, P. Perego, M. Nobile, A. Crippa and M. Molteni, Motor planning and control in autism. A kinematic analysis of preschool children, Research in Autism Spectrum Disorders 5(2) (2011), 834-842.

[22] G. Esposito, P. Venuti, F. Apicella and F. Muratori, Analysis of unsupported gait in toddlers with autism, Brain and Development 33(5) (2011), 367-373.

[23] J. Bo, C.-M. Lee, A. Colbert and B. Shen, Do children with autism spectrum disorders have motor learning difficulties?, Research in Autism Spectrum Disorders 23 (2016), 50-62.

[24] R. Downey and M.J.K. Rapport, Motor activity in children with autism: a review of current literature, Pediatric Physical Therapy 24(1) (2012), 2-20.

[25] M. Shetreat-Klein, S. Shinnar and I. Rapin, Abnormalities of joint mobility and gait in children with autism spectrum disorders, Brain and Development 36(2) (2014), 91-96.

[26] G. Dawson and R. Watling, Interventions to facilitate auditory, visual, and motor integration in autism: A review of the evidence, Journal of autism and developmental disorders 30(5) (2000), 415-421.

[27] G.T. Baranek, Efficacy of sensory and motor interventions for children with autism, Journal of autism and developmental disorders 32(5) (2002), 397-422.

[28] M. Calhoun, M. Longworth and V.L. Chester, Gait patterns in children with autism, Clinical Biomechanics 26(2) (2011), 200-206. 
[29] J.L. Cook, S.-J. Blakemore and C. Press, Atypical basic movement kinematics in autism spectrum conditions, Brain 136(9) (2013), 2816-2824.

[30] N. Papadopoulos, J. McGinley, B.J. Tonge, J.L. Bradshaw, K. Saunders and N.J. Rinehart, An investigation of upper limb motor function in high functioning autism and Asperger's disorder using a repetitive Fitts' aiming task, Research in Autism Spectrum Disorders 6(1) (2012), 286-292.

[31] M.B. Nebel, S.E. Joel, J. Muschelli, A.D. Barber, B.S. Caffo, J.J. Pekar and S.H. Mostofsky, Disruption of functional organization within the primary motor cortex in children with autism, Human brain mapping 35(2) (2014), 567-580.

[32] M. Wedyan, A. Al-Jumaily and A. Crippa, Early Diagnose of Autism Spectrum Disorder Using Machine Learning Based on Simple Upper Limb Movements, in: International Conference on Hybrid Intelligent Systems, Springer, 2018, pp. 491-500.

[33] R. Griffiths, "The ability of young children. A study in mental measurement, London: University of London Press, London, 1970.

[34] A. Barnett, A. Guzzetta, E. Mercuri, S. Henderson, L. Haataja, F. Cowan and L. Dubowitz, Can the Griffiths scales predict neuromotor and perceptual-motor impairment in term infants with neonatal encephalopathy?, Archives of disease in childhood 89(7) (2004), 637-643.

[35] A.P. Association, Diagnostic and statistical manual of mental disorders (4th Text Revision ed.). Washington, DC: Author, American Psychiatric Association, Washington, DC, 2000.

[36] C. Jarrold and J. Brock, To match or not to match? Methodological issues in autism-related research, Journal of autism and developmental disorders 34(1) (2004), 81-86.

[37] C. von Hofsten, Structuring of early reaching movements: a longitudinal study, Journal of motor behavior 23(4) (1991), 280-292.

[38] E. Thelen, D. Corbetta and J.P. Spencer, Development of reaching during the first year: role of movement speed, Journal of experimental psychology: human perception and performance 22(5) (1996), 1059.

[39] Z.M. Hira and D.F. Gillies, A review of feature selection and feature extraction methods applied on microarray data, Advances in bioinformatics 2015 (2015).

[40] I. Guyon and A. Elisseeff, An introduction to feature extraction, in: Feature extraction, Springer, 2006, pp. 1-25.

[41] P. Xanthopoulos, P.M. Pardalos and T.B. Trafalis, Linear discriminant analysis, in: Robust data mining, Springer, 2013 , pp. 27-33.

[42] W. Dwinnell and D. Sevis, LDA: Linear Discriminant Analysis, Matlab Central File Exchange 29673 (2010).

[43] S. Balakrishnama and A. Ganapathiraju, Linear discriminant analysis-a brief tutorial, Institute for Signal and information Processing 18 (1998), 1-8.

[44] S.J. Prince and J.H. Elder, Probabilistic linear discriminant analysis for inferences about identity, in: 2007 IEEE 11th International Conference on Computer Vision, IEEE, 2007, pp. 18.

[45] A.J. Bramhandkar, Discriminant analysis: applications in finance, Journal of Applied Business Research 5(2) (2011), 3741.

[46] S. Wang, D. Li, Y. Wei and H. Li, A feature selection method based on fisher's discriminant ratio for text sentiment classification, in: International Conference on Web Information Systems and Mining, Springer, 2009, pp. 88-97.
[47] P. Perner, Machine Learning and Data Mining in Pattern Recognition: 10th International Conference, MLDM 2014, St Petersburg, Russia, July 21-24, 2014, Proceedings, Springer, 2014.

[48] C.J. Burges, A tutorial on support vector machines for pattern recognition, Data mining and knowledge discovery 2(2) (1998), 121-167.

[49] W.S. Noble, What is a support vector machine?, Nature biotechnology 24(12) (2006), 1565-1567.

[50] V. Ravi, N. Naveen and M. Pandey, Hybrid classification and regression models via particle swarm optimization auto associative neural network based nonlinear PCA, International Journal of Hybrid Intelligent Systems 10(3) (2013), 137-149.

[51] A. Shmilovici, Support vector machines, in: Data mining and knowledge discovery handbook, Springer, 2009, pp. 231-247.

[52] S. Tong and D. Koller, Support vector machine active learning with applications to text classification, Journal of machine learning research 2(Nov) (2001), 45-66.

[53] D. Meyer and F.T. Wien, Support vector machines, The Interface to libsvm in package e1071 (2015), 28, in package e1071.

[54] M. Sokolova and G. Lapalme, A systematic analysis of performance measures for classification tasks, Information Processing \& Management 45(4) (2009), 427-437.

[55] C. Ferri, J. Hernández-Orallo and R. Modroiu, An experimental comparison of performance measures for classification, Pattern Recognition Letters 30(1) (2009), 27-38.

[56] R. Kohavi, A study of cross-validation and bootstrap for accuracy estimation and model selection, in: Ijcai, Vol. 14, Montreal, Canada, 1995, pp. 1137-1145.

[57] W. Zhu, N. Zeng and N. Wang, Sensitivity, specificity, accuracy, associated confidence interval and ROC analysis with practical SAS implementations, NESUG proceedings: health care and life sciences, Baltimore, Maryland 19 (2010), 1-9.

[58] F. Lauer and Y. Guermeur, MSVMpack: a multi-class support vector machine package, Journal of Machine Learning Research 12(Jul) (2011), 2293-2296.

[59] J. Weston and C. Watkins, Multi-class support vector machines, 1998.

[60] A. Vedaldi, A MATLAB wrapper of SVMstruct, 2011. 